\title{
A novel power state evaluation method for the lithium battery packs based on the improved external measurable parameter coupling model.
}

\author{
WANG, S.-L., STROE, D.-I., FERNANDEZ, C., XIONG, L.-Y., FAN, Y.-C., \\ CAO, W.
}




\title{
A novel power state evaluation method for the lithium battery packs based on the improved external measurable parameter coupling model
}

\author{
Shun-Li Wang ${ }^{\text {ab }}$, Daniel-Ioan Stroe ${ }^{\mathrm{b}}$, Carlos Fernandez ${ }^{\mathrm{c}}$, Li-Ying Xionga, Yong-Cun Fan ${ }^{\mathrm{a}}$, Wen Cao ${ }^{\mathrm{a}}$ \\ ${ }^{a}$ School of Information Engineering \& Robot Technology Used for Special Environment Key Laboratory \\ of Sichuan Province, Southwest University of Science and Technology, Mianyang 621010, China; \\ ${ }^{b}$ Department of Energy Technology, Aalborg University, Pontoppidanstraede 1119220 Aalborg East, \\ Denmark; 'School of Pharmacy and Life Sciences, Robert Gordon University, Aberdeen AB10-7GJ, UK.
}

\begin{abstract}
The power state evaluation plays a decisive influence on the safety implication of the lithium battery packs, and there is no effective online evaluation method so far due to the imbalance phenomenon among the internal connected battery cells, which cannot be abstained by the advancement of the materials and techniques. A novel power state description method is proposed in this paper by investigating the improved external parameter coupling treatment, in which the mutual relationship description is conducted by the parameter information feature decomposition together with the Bayesian sequential decision algorithm. The complicated power state evaluation model constructing problem of the coupling relationship decomposition is solved by investigating the non-convex optimization under complex working conditions for the lithium battery packs. The evidence combination is realized by introducing the information fusion strategy, according to which the multi criteria decision is realized by using the evidence theory. As can be seen from the experimental results, the voltage difference is within $10 \mathrm{mV}$ in both of the first phase and the second phase, which increases rapidly in the third phase and reaches a maximum of $120 \mathrm{mV}$. Meanwhile, its power state evaluation accuracy is $95.00 \%$ and has a good output voltage tracking effect in the complex working conditions. The power state evaluation problem can be solved by the proposed model constructing method, which is suitable for the complex battery cell combination structures and environment influences, realizing the reliable and hierarchical power state evaluation of the lithium battery packs. It provides a safety reference value and the energy management basis for the reliable power supply in the cleaner production of the power lithium battery packs.
\end{abstract}

Key words: data modeling; coupling relationship model; lithium battery pack; multi-parameter optimization; power state evaluation

Corresponding author: Shun-Li Wang. Tel: +86-15884655563. E-mail address: 497420789@qq.com.

\section{Introduction}

The power lithium battery pack plays an important role in optimizing the energy structure, ensuring the power supply and promoting the pollution reduction. It is an important technical feature and development direction in the new energy field [1], which promotes the economic and social development effectively. However, the safety problem caused by the inability of the power state has become a major hidden danger to the battery security.

The complex series-parallel combination structure is used in the lithium battery pack to break through the limitation of the cell voltage and capacity [2]. Due to the inevitable battery cell difference in the manufacturing [3] and application process, the imbalance phenomenon exist between its internal battery cells [4], making a great technical problem in the practical applications [5]. The local overheating of the lithium battery leads to the safety accidents such as spontaneous combustion and thermal imbalance between modules [6], which will bring the catastrophic consequences into society [7]. Meanwhile, the problem cannot be solved completely by the material improvement [8].

The safety accidents caused by the cell-to-cell imbalances pose a great threat to the battery security, which is the cornerstone of the social stability and economic development [9]. At this stage, the public security faces severe challenges and puts forward a major strategic demand for the science and technology [10]. The online power state evaluation is an effective means to avoid the safety accidents, making it to be another important way to improve the safety of the power lithium battery packs [11]. Under the influence of many factors such as complex working conditions, cell combination structure and environments [4], there are more interference information in the parameters [12] such as voltage, current and temperature which are measured during the working process in real-time, making the online state evaluation to be quite difficult [13]. The problem of the false alarm rate is still unable to break through [14]. Therefore, the online reliable power state 
evaluation is the key to contain the safety threat of lithium battery packs [15], which also attracts the international concern.

The reasonable application of the lithium battery pack requires the online power state evaluation to judge whether it is safe qualitatively, which provides a reference value as a basis for the control decision-making purpose of its safety management [16]. The internal parameters in the lithium battery packs cannot be detected online, such as electrolyte concentration [17], positive-negative materials and diaphragm micropore changes [18]. The parameters such as ohmic internal resistance [19], polarization resistance and polarization capacitance in the equivalent circuit model also need to be calculated indirectly through offline experiments [20]. Only the external measurable parameters, such as voltage, current, and temperature, can be measured online [21]. Therefore, the power state evaluation can only be realized by using these external measurable parameters [22]. When it is used in the power applications such as electric vehicles, aerospace and mobile base stations [12], the lithium battery pack consists of a complex combination of multiple battery cell units in series-parallel structure [23], as well as the influence of the operating and environmental conditions [24]. Because of the interference information and complex coupling relationship among the internal battery cells [25], it is difficult to characterize the working state of each battery cell directly, which makes the reliable power state evaluation to be difficult [26] and becomes a recognized worldwide problem in academia and industry [27]. Therefore, it is necessary to construct a coupling relationship model among the external measurable parameters under the influence of complex working situations [28], aiming to provide a qualitative judgment on whether the power state meets the requirements online [29]. At the same time, the power state should be evaluated accurately and make it to be safe along with the parameter acquisition time increasement [30], which provides early warning and decision-making information and becomes the research focus of the lithium battery pack security [31].

The difficulty in the coupling mechanism description is that the complex external measurable parameters have a great influence on the battery output characteristics [32], which are composed of a variety of information, such as characteristic information [33], working condition, etc. [34]. It makes the interference information exist in the original parameter sequence [35], according to which it is difficult to obtain the general time domain spectrum and realize the effective extraction of the feature information and the accurate coupling relationship of the external measurable parameters [36]. Because of the complexity and combined structure constraints [37], the detected parameter signals are ambiguous [38] along with the environmental condition influence [39] such as temperature and humidity. As a reslut, it is difficult to realize the effective online power state evaluation by the conventional methods.

The coupling measurable parameter relationship is an important part for the power state evaluation of the lithium battery pack [40]. The statistical characteristics, information redundancy representation and parameter coupling relationship from the complex working conditions can be obtained by investigating the battery cell combination structure [41]. As can be known from the simulated working condition experiments and the mathematical modeling process, the influence of the interference information is eliminated and the information is used to construct the parameter coupling relationship model [42] by conducting the power state evaluation [43]. On the one hand, the feature decomposition is divided into two types: structured and unstructured [44]. The prior information of the external measurable parameters is used to perform the Bayesian sequential decision treatment and the power state parameters can be obtained under complex working conditions [45]. The coupling relationship coordinates the decomposition by using the alternate direction multiplier [46], which reduces the computational complexity in the evaluation modeling process and corrects the coupling relationship description error [47]. Under the complex perspective information fusion, the power state evaluation model is constructed along with the volume combination structure [48], and the environmental influence on the power state evaluation process is improved by the correction knowledge base and the iterate calculation algorithm design [49], which improves the online power state evaluation accuracy effectively.

\section{Mathematical analysis}

The relationship decoupling and identification of the external measurable and coupling parameters are the key to the accurate power state evaluation of the lithium battery packs. This manuscript mainly studies how to construct the coupling relationship model between parameters under complex working conditions by investigating the parameter information feature decomposition and the Bayesian sequential decisionmaking treatment. The alternating direction multiplier is introduced to coordinate the decomposition process of the non-convex optimization model, aiming to provide the data foundation and combination for the iterate calculation process. The characteristic information of each parameter under the complex single-combination 
structure is combined and the multi-criteria decisionmaking strategy is carried out. Combined with the environmental influence, the modified strategy is studied and the grading power state evaluation is investigated online.

\subsection{Parameter coupling}

The working condition simulation experiment is carried out accordingly under different power supply scenarios to study the variation law of the external measurable parameters such as the output voltage and the temperature rise under different working conditions. Combined with it, the influence mechanism of the measured parameters can be obtained together with the working characteristics of the lithium battery pack, which provides a prior knowledge basis for the coupling relationship modeling process under complex environmental conditions. The feature decomposition is carried out for the external measurable parameter time series information, according to which the mutual influence law between these parameters can be obtained.

The charge-discharge process of the lithium battery complicates its nonlinear characteristics, so that it is especially important to estimate the remaining capacity accurately. It is especially important to establish a suitable battery performance model. The equivalent circuit model has good nonlinearity and can simulate the battery terminal voltage when the current is abrupt. Considering the electro-chemical polarization phenomenon, the RC circuit network connected in parallel with large time constant is introduced to indicate the concentration polarization. The Thevenin model is insufficient in describing the polarization characteristics of lithium battery cells. The improved circuit is proposed to increase the accuracy of the one-order RC loop, and the two-order links are simulated with better precision. As a result, the dynamic characteristics are not too complicated and the model structure is shown in Fig. 1.

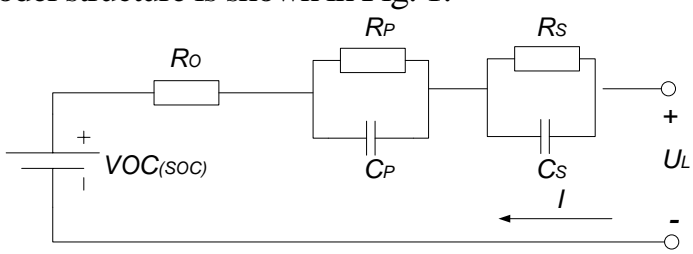

Fig. 1. The equivalent circuit model structure

According to the combined structural analysis of the lithium battery cells, the external measurable parameters can be obtained under different packing structures along with their time series characteristics. On the basis of obtaining the consistent description of the tested objects, the correlation is realized between a different battery cell combination structures and power state evaluation process. The power state evaluation is realized by investigating the multi-criteria decision-making treatment, in which the credibility calculation and evidence combination with the information fusion strategy are used to construct the power state evaluation model that is suitable for the complex single-combination structure.

As can be known from the perspective information fusion, the constraint conditions can be obtained for the prior knowledge of the power state evaluation, and the influence degree of each input parameter is analyzed and its weight is investigated for the iterate calculation process. The parameter information is fused by the combination rule, in which the model parameters and weight factors are corrected to conduct a more reliable judgment on the balance state level of the internal battery cells. The state space equation of the battery equivalent circuit model is described as shown in Equation (1).

$$
\left\{\begin{array}{l}
u=V_{O C}(S O C)-i(t) R_{O}-V_{S}-V_{L} \\
\frac{d V_{S}}{d t}=-\frac{V_{S}}{R_{S} C_{S}}+\frac{i}{C_{S}} ; \frac{d V_{L}}{d t}=-\frac{V_{L}}{R_{L} C_{L}}+\frac{i}{C_{L}}
\end{array}\right.
$$

Wherein, $T_{s}=-R_{s} C_{s}, \tau_{L}=-R_{L} C_{L}$, which represent the time response constants of the two RC parallel circuit respectively. Taking $S O C, U_{s}, U_{p}$ as the state variable and terminal voltage $U_{L}$ as the output variable, the state-space equation and the spatialized system measurement equation of the battery equivalent model can be obtained by investigating the discretization and linearization treatment as shown in Equation (2).

$$
\left\{\begin{array}{c}
{\left[\begin{array}{c}
S O C_{k+1} \\
U_{P, k+1} \\
U_{S, k+1}
\end{array}\right]=\left[\begin{array}{ccc}
1 & 0 & 0 \\
0 & 1-T / \tau_{P} & 0 \\
0 & 0 & 1-T / \tau_{S}
\end{array}\right]\left[\begin{array}{c}
S O C_{k} \\
U_{p, k} \\
U_{S, k}
\end{array}\right]+\left[\begin{array}{c}
-\varphi \eta T / Q_{n} \\
T / C_{P} \\
T / C_{S}
\end{array}\right] I+\left[\begin{array}{l}
\omega_{1, k} \\
\omega_{2, k} \\
\omega_{3, k}
\end{array}\right]} \\
U_{L, k+1}=U_{O C(S O C, k+1)}-U_{P}-U_{S}-I R_{O}+v_{k}
\end{array}\right.
$$

The mathematical rigor of the parameter coupling relationship is obtained, according to which the multifactor evaluation criteria of the designed model is used to improve the adaptability, stability and robustness of the multi-sequence coupling model. The joint solution of these time-varying signals can be obtained by using this coupling method. As the non-convex optimization model is easy to fall into the local minimum and high complexity risks, the efficient parameter coupling relationship model is explored aiming at solving this problem. The experimental data foundation and combined decision reference basis are provided for the model construction of its power state evaluation. The recursive least square method has high precision and robustness advantages for the parameter identification. As a result, it is used to identify the parameters of $R_{o}, R_{p}, R_{s}$, $C_{p}$ and $C_{s}$ in the equivalent circuit model, according to which the calculation formula can be obtained as shown in Equation (3).

$$
U_{o C}-U_{O}=u\left[R_{P} /\left(R_{P} C_{P} s+1\right)+R_{e}+R_{S} /\left(R_{S} C_{S} s+1\right)\right]
$$


Combined with the sequential probability ratio test method, the feature information screening algorithm is constructed. In order to build the coupling relationship model with the feature information discriminating process, the priori information of the external measurable signal is used and the non-convex optimization is investigated by alternating the direction multiplier method, which is used to reduce the computational complexity in the model solving process. The calculation error can be corrected simultaneously, which is caused by the discrete signal sampling and complex operating conditions. The alternating direction multiplier is introduced to coordinate the decomposition of the model solution process, according to which the qualitative identification is realized for its completed model parameters. The linearization treatment is performed using the above formula, the parameter relationship of which is $y(k)=U_{O C^{-}}$ $U_{O}$. Then, the parameter relationship equations can be obtained as shown in Equation (4).

$$
\begin{aligned}
& y(k)=-k_{1} y(k-1)-k_{2} y(k-2)+k_{3} u(k) \\
& +k_{4} u(k-1)+k_{5} u(k-2)
\end{aligned}
$$

The parameter relationship in the above equation is shown in Equation (5).

$$
\left\{\begin{array}{l}
\tau_{P}=R_{P} C_{P}, \tau_{S}=R_{S} C_{S} \\
R_{Q} \tau_{P} \tau_{S}=\left(k_{5}-k_{4}+k_{3}\right) T^{2} /\left[4\left(k_{2}+k_{1}+1\right)\right] \\
R_{O} \tau_{P}+R_{S} \tau_{S}+R_{P} \tau_{S}+R_{S} \tau_{P}=T\left(k_{3}-k_{5}\right) /\left(k_{2}+k_{1}+1\right) \\
R_{O}+R_{S}+R_{P}=\left(k_{5}+k_{4}+k_{3}\right) /\left(k_{2}+k_{1}+1\right) \\
\tau_{P} \tau_{S}=\left(k_{2}-k_{1}+1\right) T^{2} /\left[4\left(k_{2}+k_{1}+1\right)\right] \\
\tau_{P}+\tau_{S}=T\left(1-k_{2}\right) /\left(k_{2}+k_{1}+1\right)
\end{array}\right.
$$

In the above Equation, $T$ is the sampling time period and its least square expression is shown in Equation (6).

$$
\left\{\begin{array}{l}
y(k)=H^{T} \theta+e(k) \\
\theta=\left[k_{1}, k_{2}, k_{3}, k_{4}, k_{5}\right]^{T}
\end{array}\right.
$$

Wherein, $e(k)$ is the error function, in which the Bayesian sequential decision treatment is introduced into the calculation process to construct its coupling relationship model. It is difficult to extract the feature information under complex working conditions, so the discriminating strategy is introduced for the time-varying signal of its external measurable parameters. The prior knowledge constraints are obtained in the model parameter identification process, in which the coupling relationship is characterized by optimizing the combination description. The least square recursion formula can be obtained as shown in Equation (7).

$$
\left\{\begin{array}{l}
H^{T}(k)=[-y(k-1)-y(k-2) u(k) u(k-1) u(k-2)] \\
\theta(k)=\theta(k-1)+K(k)\left[y(k)-H^{T}(k) \theta(k-1)\right] \\
K(k)=P(k) H(k)\left[H^{T}(k) P(k) H(k)+1\right]^{-1} \\
P(k)=\left[\operatorname{eye}(5)-K(k) H^{T}(k) P(k-1)\right]
\end{array}\right.
$$

The environmental affecting factors are obtained in the power state evaluation process through the experimental research, in which the complex environmental condition influence can be characterized by the power state evaluation. The parameter correlation treatment is investigated between the influencing factors and the evaluation process, according to which the knowledge base of the power state evaluation can be built under complex environmental conditions. The optimal expression and parametric eigenvalue description methods are introduced for the time-varying signals, according to which an effective correction algorithm is designed to eliminate the evaluation errors introduced by the environmental conditions and further improves the calculation speed and accuracy of the power state evaluation.

\subsection{Interference information}

The type of the interference information is analyzed by investigating the complex working condition analysis, and the coupling relationship model of the external measurable parameters can be constructed by combining the information feature decomposition. The typical working conditions are analyzed, such as electric vehicles, aviation and mobile base stations. Then, the types of the interference information are studied that can be introduced in the parameter detection, filtering and state evaluation process, which determines the statistical model of the interference information: impulse, Rayleigh distributed and Gaussian signals.

The regular term in the model consists of two parts: one is the regularization term reflecting the coupling relationship between the external measurable parameters, and the other is the decision item reflecting the useful feature information to eliminate the interference. The Sequential Probability Ratio Test (SPRT) is conducted to solve the non-convex optimization and investigate the relation model. Combined with feature information discriminating algorithm of the external measurable parameters, it is used to optimize the dynamic description of the coupling relationship and suppress the influence of the working conditions. The range values are expressed for the discrete random variable $X$ as shown in Equation (8).

$$
X=\left\{\begin{array}{lllll}
1 & 2 & \cdots & k-1 & k
\end{array}\right\}
$$

Each value in Equation (8) corresponds to the fault condition of the system time point $k$, and the output of the diagnostic network is a probability distribution of the random variable $X$. Considering the principle that the various faults are diagnosed by the SPRT algorithm, a type of fault $f(k)$ is taken as the convenience description example by considering the hypothesis test model at the time point $t$. The log likelihood probability ratio statistic at time point $t+1$ can be established as shown in Equation (9). 


$$
L R_{t+l}\left(X_{t}, \cdots, X_{t+l}\right)=\sum_{t}^{t+l} \ln \frac{P_{k}\left(X_{i}\right)}{P_{0}\left(X_{i}\right)}
$$

By conducting the feature decomposition, the external measurable parameter signal is decomposed into three parts: feature information data fidelity, structured and unstructured items. Among them, the feature information can be obtained through the offline experiments, and the structural items are obtained by means of the analytical modeling and signal fitting treatment. Then, the unstructured items are eliminated by the signal transformation by representing the measurement noise [50]. According to the coupling problem between these external measurable parameters, the variation and coupling law of each parameters are analyzed [51], which is shown in Equation (10).

$$
\gamma=\inf \left\{l \mid l \geq 1, L R_{t+l} \notin\left[\ln T\left(H_{0}\right), \ln T\left(H_{1}\right)\right]\right\}
$$

Aiming at the coupling problem between the external measurable parameters of lithium battery packs under complex working conditions, a parameter coupling relationship model is built by conducting the signal feature decomposition and Bayesian sequential decisionmaking treatment. Using the prior information of the parameter signal under the typical working conditions, the coupling relationship between the external measurable time-varying parameters can be decomposed into two types: structured and unstructured.

The structured information is used to characterize the coupling relationship between the external measurable parameters hidden in the working condition signals. According to the influence relationship of the working feature information, the interference linear/nonlinear and additive/multiplicative types can be determined that are introduced by the complex working conditions. Then, the feature information screening decision items are constructed by using the prior interference information to realize its effective feature extraction. The recursive formula of which is shown in Equation (11).

$$
L R_{t+l}=L R_{t+l-1}+\ln \frac{P_{k}\left(X_{t+l}\right)}{P_{0}\left(X_{t+l}\right)}
$$

Furthermore, the symbol $P_{F}$ can be used to indicate the allowed false alarm probability, according to which $H_{0}$ is established instead of being denied. $P_{M}$ indicates the allowable leakage alarm probability, according to which $H_{1}$ is established instead of accepting $H_{0}$. And then, the SPRT threshold is obtained as shown in Equation (12).

$$
\left\{\begin{array}{l}
T\left(H_{0}\right)=\frac{P_{M}}{1-P_{F}} \\
T\left(H_{1}\right)=\frac{1-P_{M}}{P_{F}}
\end{array}\right.
$$

The multi-criteria decision-making and information fusion strategies are introduced to solve the hierarchical power state evaluation problem because of the complex battery cell combination structure and environmental conditions. Combining the advantages of the evidence theory and the information fusion, the power state evaluation accuracy is improved for the lithium battery packs. This method is made up for the defect that the power state evaluation efficiency and the false alarm rate do not meet the requirements by using the traditional state evaluation method, which will bring new solutions and techniques to the safety guarantee of the lithium battery pack inevitably. The mathematical description of the influencing factors can be established by means of the experimental modeling treatment, and the correction algorithm is constructed to eliminate the power state evaluation error caused by the environmental condition factors, which is used to improve its evaluation accuracy.

According to the characteristic information, the timedomain waveform shape is invariant under the expansion transformation, and the observed time-varying signal sequence $\Phi(t)$ is decomposed into a mixed superposition in the coupling relationship model. The delay-expanded copy of the feature information data fidelity term $\varphi(t)$, the structured term $\psi(t)$, and the unstructured term $\eta(t)$ can be obtained over a finite time period of $[0, T]$, such as Linear, nonlinear, additive, multiplicative and so on. The calculation process of which is shown in Equation (13).

$$
\left\{\begin{aligned}
\Phi(t) & =f\left\{V_{i}(t), I_{i}(t), T_{i}(t), \cdots\right\}, i=1,2, \cdots, N \\
& =\sum_{i=1}^{I^{u}} \sum_{j=1}^{M} u_{k_{u}} \varphi\left(t-\tau_{k_{u}}^{u}\right) \oplus \sum_{i=1}^{I^{u}} \sum_{j=1}^{M} v_{k_{v}} \psi\left(t-\tau_{k_{v}}^{v}\right) \oplus \eta(t) \\
k_{u}= & (i-1) I^{u}+j, k_{v}=(i-1) I^{v}+j
\end{aligned}\right.
$$

Among them, $\Phi(t)$ is a time-varying parameter, which is also the coupling relationship description matrix of the lithium battery pack. The function $\left.f{ }^{*}\right\}$ is the relational model, in which $V_{i}(t)$ is the voltage of the $i$-th single battery cell and $I_{i}(t)$ indicates the current flowing through the $i$-th single battery cell at time point $t$. In addition, And $T_{i}(t)$ indicates the temperature value of the $i$-th single battery cell at time point $t$ corresponding to the feature information. $k$ is the amplitude corresponding to the feature information, and $k_{v}$ is the number of the feature information within the structured items.

\subsection{Bayesian sequential decision}

Aiming at the influence of complex working conditions on the coupling relationship model, the screening process is added to achieve the effective of parameter feature information extraction based on the Bayesian Sequential Decision (BSD) treatment [52]. The interference superposition type is analyzed for the typical working conditions, such as linear-nonlinear, additivemultiplicative and so on. The influence relationship of the working situation is obtained on the characteristic information, which is then determined by its statistical 
model, such as impulse, Rayleigh distribution and Gaussian signals [53]. According to the BSD method, the characteristic information discriminating model is constructed for the lithium battery packs, and the coupling relationship model is integrated into the regularization term to improve the feature information representation accuracy, thus improving the coupling relationship description accuracy and efficiency. According to the fusion decision of the state evaluation purpose, the structure of the power state evaluation system is obtained as shown in Fig. 2.

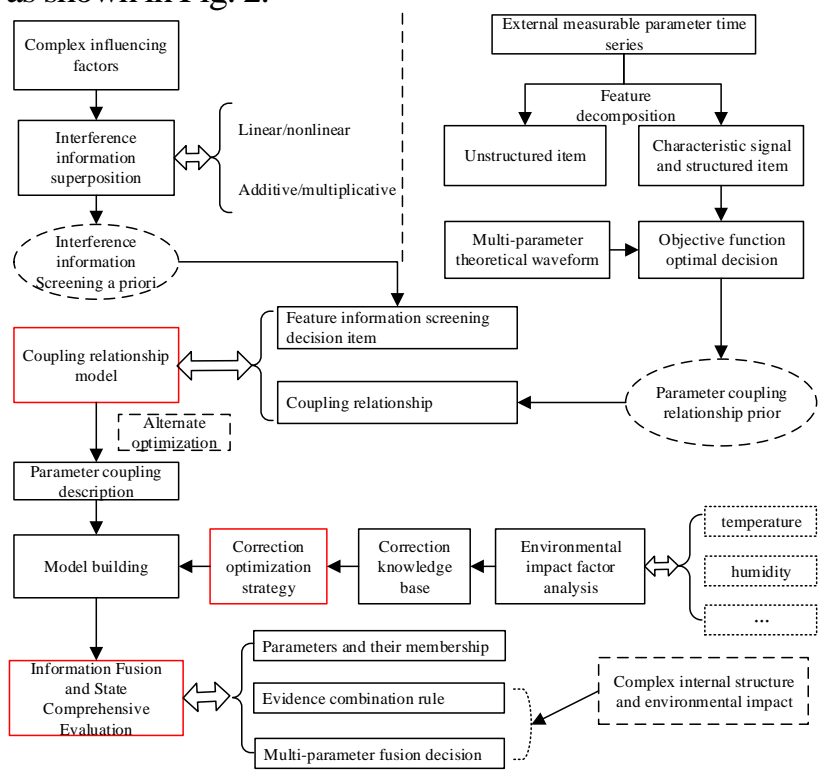

Fig. 2. The power state evaluation structure

According to the structural framework of the established parameter coupling relationship model, the regularized term composition structure is determined and the coupling relationship model is built by using the SPRT method. The form of the data fidelity term depends on the statistical signal properties, while the regularization term consists of two parts: one is the regularization term that reflects the signal coupling relationship characteristics, and the other is the decision item that reflects the parameter feature information. Since the coupling relationship model contains the feature information in the regularization term, the solution of the model parameters degenerates into the non-convex optimization process [19]. As a result, it is very difficult to solve the nonconvex optimization problem directly, which not only consumes a lot of computing resources but also easily falls into the local extremum risks.

The main calculation parameters are initialized to solve this problem, and the interaction is considered in the group working process of the internal connected battery cells [54]. The comprehensive power state and measurement values are obtained for the battery packs, which are used as the main basis of the evaluation process. According to the comprehensive value calculation of the battery cells and the application of the best priority search algorithm, the hierarchical description is determined in the power state evaluation process. The calculation process of the cell balance degree $C F\left(\varphi_{i}(t)\right)$ is shown in Equation (14).

$$
\left\{\begin{array}{l}
C F\left(\varphi_{i}(t)\right)=\frac{C F_{a}\left(\varphi_{i}(t)\right)+C F_{b}\left(\varphi_{i}(t)\right)}{1-\min \left(\left|C F_{a}\left(\varphi_{i}(t)\right)\right|,\left|C F_{b}\left(\varphi_{i}(t)\right)\right|\right)} \\
C F_{a}\left(\varphi_{i}(t)\right)=C F\left(\varphi_{i}(t), \alpha_{i}\right) \times \max \left\{0, C F\left(\alpha_{i}\right)\right\} \\
C F_{b}\left(\varphi_{i}(t)\right)=C F\left(\varphi_{i}(t), \beta_{i}\right) \times \max \left\{0, C F\left(\beta_{i}\right)\right\}
\end{array}\right.
$$

As can be seen in Equation (14), $C F(x, y)$ represents the confidence factor that is obtained according to the inference rule, and $C F_{a}\left(\varphi_{\mathrm{i}}(\mathrm{t})\right)$ and $C F_{b}\left(\varphi_{i}(t)\right)$ represent the pair balance parameter $\varphi$ respectively for the $i$-th battery cell in the packs, that is, the influence degree of the state weight function. The power state evaluation is investigated by the model parameters and weighting factors, and then the evaluation result difference are explored under different working modes to improve the evaluation accuracy. Furthermore, the experimental samples of different lithium battery packs are established, which are used to analyze the working process changes and evaluation results under different initial conditions. By incorporating the influencing factors into the power state evaluation process, the model parameters and weighting factors are corrected in the power state evaluation model. Furthermore, through multi-evidence source information fusion, the hypothesis set is reduced gradually according to the evidence accumulation, in which the inference decision process is realized by the evidence combination rule as shown in Equation (15).

$$
\left\{\begin{array}{l}
m_{1} \oplus m_{2}(Z)=\frac{\sum_{X \cap Y=Z} m_{1}(X) m_{2}(Y)}{1-K} \\
K=\sum_{X \cap Y=\varnothing} m_{1}(X) m_{2}(Y)
\end{array}\right.
$$

Wherein, $m_{1}(X)$ and $m_{2}(X)$ are the basic credibility functions of $X$ and $Y$, respectively. According to the power state evaluation framework based on the evidence theory, the factor analysis is conducted and the index system is established. And then, the influencing factors are determined, which are all subordinated to the functional Gaussian membership calculation framework to gain the degree of different evaluation levels. As the basic probability distribution is required by the DS evidence theory, the fusion weight distribution matrix is introduced. Then, the evidence combination rule selection is used to combine multiple membership functions to realize the power state classification.

Aiming at the fuzziness problem in the fusion process of external measurable parameter information under the complex internal battery cell combination structure, the 
multi-criteria decision method is introduced into the power state evaluation model of the lithium battery pack. The Dempster Shafer (DS) evidence theory is used to deal with the complex cell combination structure problems, in which the reasoning treatment is investigated to describe the power state evaluation level, so that the evaluation results can better reflect the actual state situation for each single battery cells [55]. The data correlation relationship is obtained by predicting the sampled signal, and the associated membership degree is calculated by the processing of the feature information between the sample points at the same time. It is then converted into the basic probability assignment in the evidence theory, according to which the evidence information is combined with the real-time detected parameters to obtain the time varying state values [56]. Meanwhile, the correlation result is obtained according to the association judgment of the sample points in the continuous duration process.

\subsection{UKF-EKF composite calculation}

The Kalman Filter (KF) algorithm is mainly used to predict and filter the state variables in the linear systems[57], which is not applicable for the nonlinear equations, and promotes its further development [58]. Combined the working situation analysis with the optimization description method, the model design theory is improved and optimized. Combining the impact analysis of different working conditions, the modeling and improvement methods are explored. Based on the combination of composition and structural changes [59], the model structure is modified to achieve an accurate description for the coupling treatment of the operating characteristics and parameters. The coupling expression is analyzed by using the EKF and UKF algorithms, which are emerged as the main idea. As a result, the nonlinear state equation is approximated linearly, and then the linear $\mathrm{KF}$ module is used for the power state estimation. Combined with the previous EKF-based calculation process, the UKF-based estimation is investigated by taking the test results into the correction stage. Therefore, the relative $\mathrm{UKF}$ and $\mathrm{EKF}$ algorithms require high initial state relatively, in which the state equation is described as shown in Equation (16).

$$
x_{k+1}=f\left(x_{k}, u_{k}\right)+w_{k}
$$

When the given initial value and the true initial value differ greatly, the EKF-based calculation converges slowly or does not converge. Combined with the EKFbased calculation result, the UKF algorithm performs two matrix decompositions in each iteration step, which calculates multiple sigma points with more computational complexity. Therefore, both of the EKF and UKF algorithms have special advantages for the real-time online power state evaluation, according to which the
UKF-EKF combined method is proposed for the SOC estimation method. The measurement equation is described as shown in Equation (17).

$$
y_{k}=g\left(x_{k}, u_{k}\right)+v_{k}
$$

As can be known from Equation (17), the initialized state variable and the error covariance matrix can be calculated directly when $k=0$, and the sum of $2 n+1$ points can be obtained as well. The Thresh loop performs according to the following steps and the Thresh is the threshold, which affects the prediction result obviously and its selection range is generally set as a varying value between 50 and 100. The equation for calculating the predicted state variable is shown in Equation (18).

$$
\left\{\begin{array}{l}
\hat{x}_{k}^{-}=\sum_{i=1}^{N_{i}} W_{i} f\left(\xi_{i}, u_{k-1}\right) \\
\xi_{i}=S \gamma_{i}+\hat{x}_{k-1}^{+} ; P_{k-1}^{+}=S S^{T}
\end{array}\right.
$$

In the case of a regularization term, the decision item is enabled for the feature information, and the overall power state evaluation problem is transformed into a local optimization problem of finding the feature information in the time series signals. The convex and local optimization problems are solved by the typical gradient optimization and fast search methods. Then, the UKF algorithm is used to estimate the initial state and solve the convergence problem. Meanwhile, the EKF algorithm is used for the online estimation in real-time to reduce the computational complexity, in which the covariance error is calculated according to Equation (19).

$$
P_{K}^{-}=\sum_{i=1}^{N_{p}} W_{i}\left[f\left(\xi_{i}, u_{k-1}\right)-\hat{x}_{k}^{-}\right]\left[f\left(\xi_{i}, u_{k-1}\right)-\hat{x}_{k}^{-}\right]^{T}+p_{W}
$$

The effects of environmental conditions on the power state evaluation are studied experimentally, in which the state data correction is constructed to describe the influence of the environmental conditions. Different factors affect the parameter characteristics, such as environmental condition differences, sampling fluctuations, and working condition changes (land, deep sea, high altitude, etc.). Furthermore, The Kalman gain is obtained as shown in Equation (20).

$$
\left\{\begin{array}{l}
P_{k}^{-}=\$ \xi^{6} ; \xi_{i}=\$ q_{i}+\hat{x}_{k}^{-} \\
\hat{y}_{k}=\sum_{i=1}^{N_{p}} W_{i} h\left[f\left(\xi_{i}, u_{k}\right)\right] \\
P_{y y}=\sum_{i=1}^{N_{p}} W_{i}\left[h\left(\xi_{i}, u_{k}\right)-\hat{y}_{k}\right]\left[h\left(\xi_{i}, u_{k}\right)-\hat{y}_{k}\right]^{T} \\
P_{x y}=\sum_{i=1}^{N_{p}} W_{i}\left(\xi_{i}-\hat{x}_{k}^{-}\right)\left[h\left(\xi_{i}, u_{k}\right)-\hat{y}_{k}\right]^{T} \\
L_{k}=P_{x y}\left(P_{y y}+P_{v}\right)^{-1}
\end{array}\right.
$$

In order to further reduce the measurable deterministic influence of the operating environmental factors, it is 
supposed to obtain the influence law of the environmental conditions through the experimental research and data training methods. The mathematical description is established for the influence relationship of the environmental conditions, such as temperature and humidity. The corrected state prediction step is shown in Equation (21).

$$
\hat{x}_{k}^{+}=\hat{x}_{k}^{-}+L_{k}\left(y_{k}-\hat{y}_{k}\right)
$$

The non-convex optimization problem is solved by the alternating direction multiplier, which is translated into two sub-optimization problems: convex optimization and local optimization. In the case that the fixed feature information discriminates the regularization term, the solution process can extract the mutual coupling relationship from the external measurable parameter in time series. And then, the problem is degenerated into a convex optimization process by using the time-varying vectors. The repeated experiment is conducted to obtain the influence law of the environmental conditions under the specific working scenarios, according to which the state data correction knowledge base is established and the error covariance is corrected as shown in Equation (22).

$$
P_{K}^{+}=P_{K}^{-}-L_{k} P_{x y}^{T}
$$

The correction strategy is selected according to the ambient temperature and humidity conditions in the power state evaluation process, in which the actual correction amount of the power state evaluation is calculated by conducting the mathematical description of the environmental influence relationship. As for $k$ equaling to Thresh $+1 \sim$ End, the following iterate calculation steps can be investigated accordingly, and the environmental condition influence law is analyzed to obtain the correction strategy. Combined with the application of the multivariate parameter estimation theory, the power state evaluation model structure is optimized to improve the environmental applicability of the evaluation results. And then, the state variables can be forecasted according to Equation (23).

$$
\hat{x}_{k}^{-}=f\left(\hat{x}_{k-1}^{+}, u_{k-1}\right)
$$

The influence on the evaluation effect is analyzed for the complex environmental conditions, according to which the influencing factor correction strategy is obtained. The theoretical and experimental research is conducted on the influencing factors such as chargedischarge current, temperature, humidity change and selfdischarge rate, according to which the error covariance can be obtained by Equation (24).

$$
P_{k}^{-}=\hat{A}_{k-1} P_{k-1}^{+} \hat{A}_{k-1}^{T}+P_{w}
$$

The multi-parameter estimation theory is introduced to realize the model parameter identification, in which a mathematical description of the working characteristics can be obtained for the lithium battery packs under different environmental conditions, in which the Kalman can be gained by Equation (25).

$$
L_{k}=P_{k}^{-} \hat{C}_{k}^{T}\left[\hat{C}_{k} P_{k}^{-} \hat{C}_{k}^{T}+P_{v}\right]^{-1}
$$

According to the correlation and multivariate nonlinear parameter identification, the dynamic description of the coupling relationship is realized between the external measurable parameters. Considering the working characteristics of the lithium battery pack, the coupling relationship description effect is studied between its parameters. The corrected state prediction is obtained as shown in Equation (26).

$$
\left\{\begin{array}{l}
\hat{y}_{k}=g\left(\hat{x}_{k}^{-}, u_{k}\right) \\
\hat{x}_{k}^{+}=\hat{x}_{k}^{-}+L_{k}\left(y_{k}-\hat{y}_{k}\right)
\end{array}\right.
$$

The multi-linear estimation is investigated to study the parameter identification method of the time varying model parameters [60]. Based on the experimental exploration together with its improved methods, the coefficient verification is completed, together with its error model parameter identification. And then, the accuracy and the computational complexity are coordinated, which lays a foundation for the critical breakthrough of the power state evaluation, in which the covariance error can be corrected by Equation (27).

$$
P_{k}^{+}=\left(1-L_{k} \hat{C}_{k}\right) P_{k}^{-}
$$

The environmental condition change influence is considered in the evaluation results, and the revised strategy is proposed to realize the optimization of the power state evaluation model structure.

\section{Experimental analysis}

The variation law of the lithium battery pack is studied under different working conditions, together with the battery voltage and the unbalanced state between its internal connected battery cells. The performance of the lithium battery pack is studied through the experiments, in which the power state provides a theoretical basis, and further expresses the characteristics of the group working lithium battery cells, which provides a basis for the safety protection of the lithium battery pack.

\subsection{Experimental platform design}

In the experiments, 7 sets of series-connected lithium cobalt oxide lithium battery cells with a rated capacity of $4 \mathrm{Ah}$ and a rated voltage of $3.7 \mathrm{~V}$ are selected as the experimental samples. The lithium battery pack is tested by using the battery test system, and its working voltage and remaining power state data can be collected by using the experimental platform as shown in Fig. 3. 


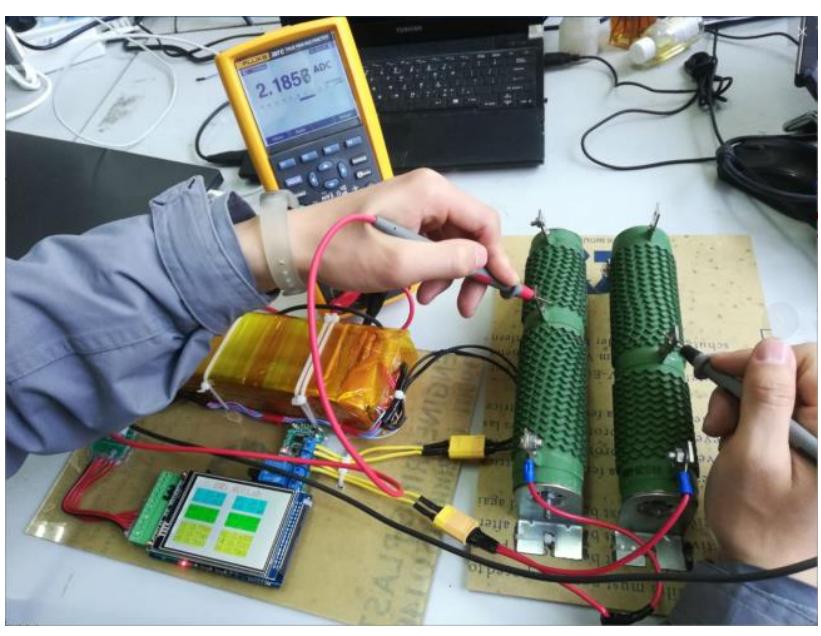

Fig. 3. The experimental platform

This experiment simulates the working state of the lithium battery pack during the flight. The lithium battery cells used in this experiment are mainly tested for the instrument inspection, ignition and emergency output on the aircraft, which also supplements the battery through the engine during the flight. The capacity of the lithium battery is maintained within a predetermined range, according to which three different working conditions are designed and the parameter settings are shown in Tab. 1.

Tab.1 Parameter settings under different conditions

\begin{tabular}{llllll}
\hline Condition & $\mathrm{a}(\mathrm{s})$ & $\mathrm{b}(\mathrm{s})$ & $\mathrm{c}(\mathrm{s})$ & $\mathrm{d}(\mathrm{s})$ & $\mathrm{e}(\mathrm{s})$ \\
\hline 1 & 60 & 5 & 20 & 10 & 50 \\
\hline 2 & 30 & 5 & 20 & 10 & 80 \\
\hline 3 & 100 & 5 & 20 & 10 & 200
\end{tabular}

Wherein, $\mathrm{a}$ is the time to check the control sub-systems, $\mathrm{b}$ is the aircraft ignition discharge time, $\mathrm{c}$ is the supplementary power time, $\mathrm{d}$ is the time for the simulated self-discharge, and e is the emergency output time. And then, the experimental steps designed are shown as follows.

(1) It should be determined whether the cell voltage and the terminal voltage of the battery pack are greater than the minimum voltage of $3 \mathrm{~V}$ and $21 \mathrm{~V}$. If it satisfies this condition, it should turn to step (2), otherwise it should jump to step (10).

(2) The battery cells should be set aside for 10 s to proceed to step (3).

(3) The experiment should amplify the instrument with 0.3C discharging current rate for one second, aiming to judge whether the cell voltage and total terminal voltage are greater than the minimum voltage levels. If it satisfies the condition, it will turn to step (4), otherwise it should jump to step (10).

(4) The experiment simulates the aircraft ignition with $0.6 \mathrm{C}$ discharging current rate for $\mathrm{b}$ seconds, and then it should be judged whether the cell voltage and the terminal voltage are greater than the minimum voltage. If it satisfies the condition, it will turn to step (5), otherwise it will jump to step (10).

(5) After charging with $0.1 \mathrm{C}$ for $\mathrm{c}$ seconds, the analog power is added and then turn to step (6).

(6) The self-discharge of the lithium battery is simulated by discharging at $0.01 \mathrm{C}$ for $d$ seconds, and then it proceeds to step (7).

(7) After charging with $0.1 \mathrm{C}$ current for $\mathrm{c}$ seconds, the experimental process should enter the 8th step.

(8) The emergency output is simulated with 1C discharging current rate for few seconds, and then it should be judged whether the cell voltage and terminal voltage are greater than the minimum voltages. If it satisfies the condition, it turns to enter step (9), otherwise it jumps to step (10).

(9) The steps (2) (8) should be repeated until it jumps to step (10).

(10) End.

\subsection{Different magnifications test}

Seven 4Ah aviation lithium battery cells are connected in series, which are charged and discharged by using the charge-discharge current rates of $0.2,0.3,0.5,1,1.2$ respectively. Based on the test data, the time varying voltage data during charging and discharging process can be plotted at different current rates. The battery voltage changing law can be obtained along with the magnification during the charging process, according to which the variation curve is shown in Fig. 4.

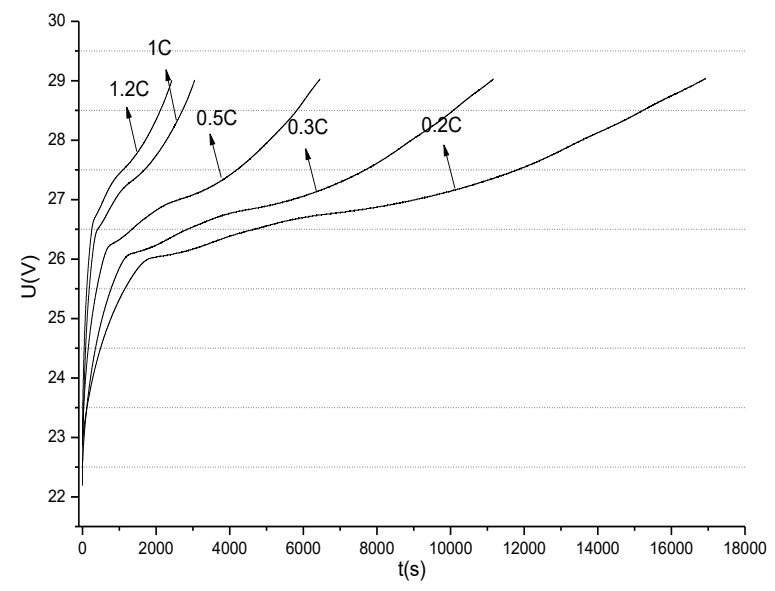

Fig. 4. Charging Voltage changes with magnification

By analyzing the voltage variation curve in Fig. 4, it can be known that the voltage of the charging battery platform region changes greatly under the high-rate discharging current conditions under the same constant-current charging precondition treatment. The voltage change of the lithium battery can be roughly divided into the following two stages. (1) The battery voltage rises rapidly 
at the initial stage of the charging process. (2) The battery voltage rises slowly after a certain period of the charging treatment. After that, the different discharging current rate maintenance experiments are investigated, according to which the discharging characteristics are obtained as shown in Fig. 5.

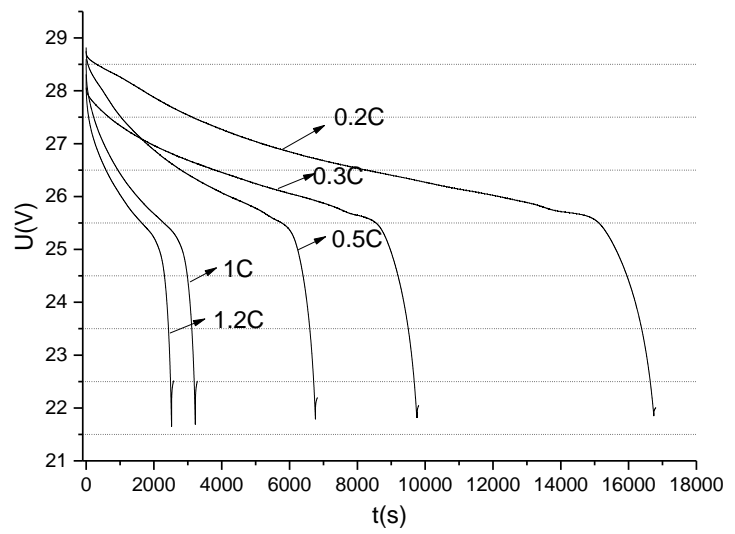

Fig. 5. Discharging voltage change with magnification

As can be known from the voltage curve of Fig. 5, the voltage of the discharging platform area changes greatly under high-rate discharging conditions. However, a sudden voltage drop occurs at the beginning of the discharging process with $0.3 \mathrm{C}$ current rate. The voltage change of the lithium battery can be roughly divided into the following three stages. (1) The battery voltage drops rapidly at the initial stage of the discharge process. (2) The battery voltage reduction rate is relatively gentle, which enters the platform area after a certain time period of discharge. (3) The battery voltage drop rate is faster than the initial stage after a certain time period of discharge.

\subsection{Voltage variation Influence}

The terminal voltage of the lithium battery pack varies along as time goes by under different operating conditions, which drops rapidly at the initial stage of the operating conditions, slowly in the middle section, and rapidly at the end stage. However, due to the experimental frequency difference in the various operating conditions, the voltage curve of the battery pack exhibits different characteristics over time. Comparing the battery voltage curve towards time under these three working conditions, the voltage varying rate is bigger when the operating condition change rate is greater, the experimental result of which can be obtained as shown in Fig. 6.

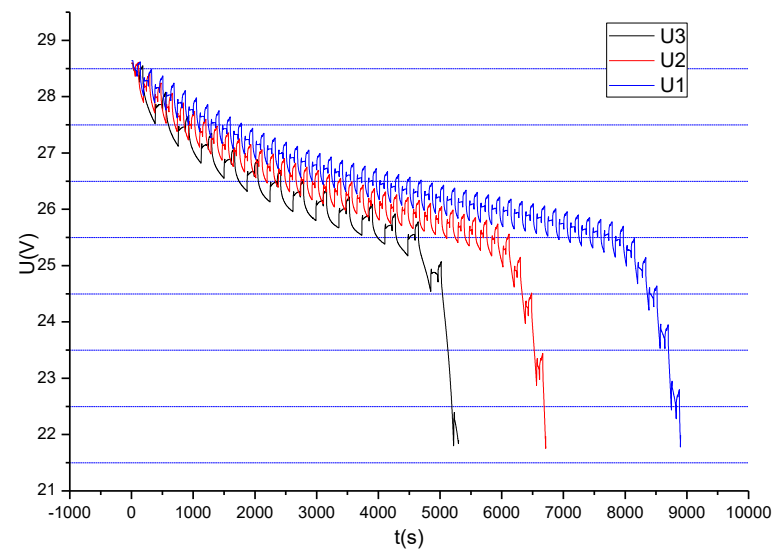

Fig. 6. Packing voltage change under different conditions

As shown in Fig. 6, U1, U2, and U3 represent the battery voltage curves towards time under the working conditions of 1, 2 and 3 respectively.

\subsection{Working state filtering result}

In order to verify the feasibility of the above power state evaluation algorithm, the mathematical iterate calculation model is established to realize the difficult on-line power state evaluation between cells under the influence of the complex working conditions, according to which the multi-criteria decision making treatment of the evidence theory is proposed. Based on the characteristic exploration of the different working conditions, the coupling relationship model related to the parameter acquisition time is established, by which the non-convex optimization problem is solved using the alternating direction multiplier. The experimental data is obtained by the test, which can be brought into the model as the input parameters together with the constant-current working conditions. And then, the simulation results are obtained and analyzed, which are shown in Fig. 7.

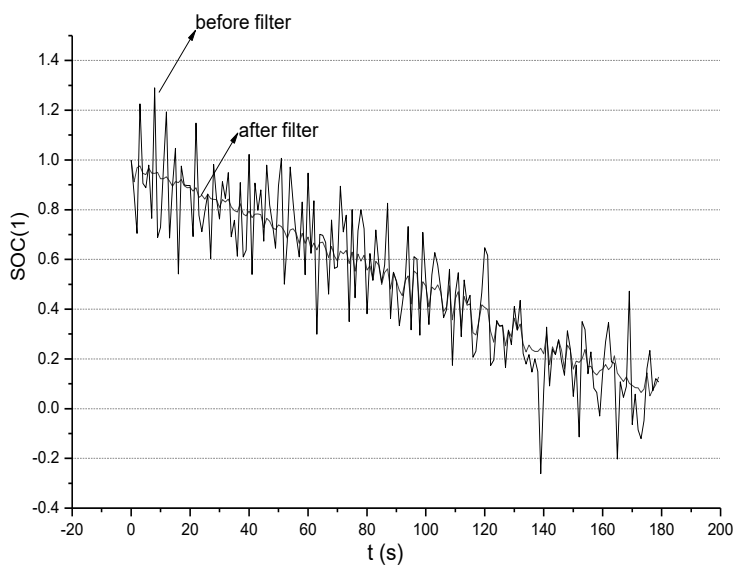

Fig. 7. Working state filtering result 
As can be known from Fig. 7, one curve is obtained by filtering with the combined UKF-EKF algorithm and the other is not filtered, which has better experimental results obviously. Therefore, the established power state estimation model can realize the real-time working state monitoring, in which the estimation error is less than 0.05 . Based on the second-order Thevenin equivalent circuit, the working state and output characteristics of the lithium battery pack can be described effectively, in which the HPPC test is investigated by using the above parameter identification treatment and the recursive least square method. The charging characteristics are also obtained through the charge-discharge test of the aviation lithium battery pack under different magnifications, based on which the joint UKF-EKF algorithm is proposed for the online power state evaluation. The comparison between the simulation and the experimental test results show that the estimation accuracy is higher than $95 \%$ under the scheme, which has high precision and tracking advantages. The key to use the battery rationally and improve its life span is to estimate its power state accurately, the estimated effect of which will directly determine the power and economy of the packing lithium battery cells.

Through the experiment of aeronautical lithium battery pack under different working conditions, the battery voltage curve and its variation law can be obtained [61]. The characteristics of aeronautical lithium battery pack under different working conditions are analyzed and compared with the related references [62]. The following conclusions can be drawn accordingly. (1) The voltage variation of the aviation lithium battery pack is large under the working conditions, and the more frequent the change of the working condition, the greater the battery voltage change rate is [63]. (2) The individual battery cell voltage is almost the same as the voltage change of the battery pack. The cell voltage difference increases rapidly in the third stage of the voltage drop [64]. The imbalance state between the battery cells will be deeper when the discharge depth is greater in a single charge-discharge cycle. When, the inconsistency is manifested, the battery should be used to avoid long-time discharge at a large rate. On the other hand, the lithium battery pack needs to be maintained regularly and a battery equalization system should be used to realize the balance treatment under complicated conditions.

\subsection{Battery cell inconsistency analysis}

During the working time period of the lithium battery pack, the voltage change is still divided into three stages. According to the standard experimental process, the SOC is reduced from $100 \%$ to $80 \%$ at the first stage and the voltage drops rapidly in this stage. The second stage is reduced from $80 \%$ to $30 \%$, in which the voltage curve of this stage is relatively flat. The SOC value is set from $30 \%$ to 0 for the third stage, which is also the voltage decline phase. Because the voltage difference between the internal connected battery cells of the lithium battery pack is the main parameter for characterizing the inconsistency of the internal cells, the service life of the battery pack and the full utilization of its power can be affected seriously. Therefore, it is especially important to study the inconsistency of the lithium battery under the working conditions. As a result, the absolute value of the voltage difference between the battery cells and the mean voltage value are used to characterize the inconsistency degree of the voltage between the lithium battery cells. Through the data collected by the experiment, the cell voltage variation graph towards time under these three working conditions and the curve of the voltage difference between the battery cells are plotted, which are shown in Fig. 8.
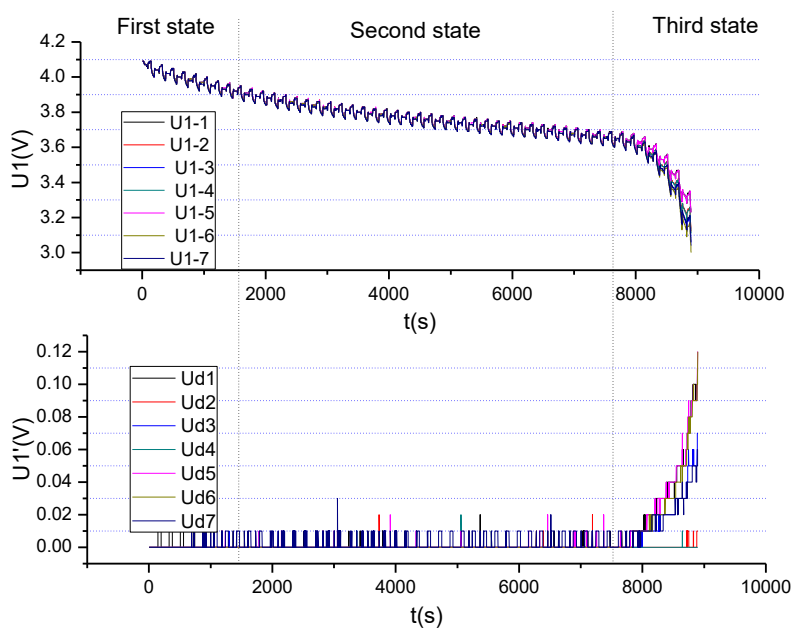

Fig. 8. Voltage difference under working condition 1

The signals of U1-1, U1-2, U1-3, U1-4, U1-5, U1-6, U1-7 in Fig. 8 represent the voltage of the 7 single cells under the working condition 1 . The Ud1, Ud2, Ud3, Ud4, $\mathrm{Ud5}$, Ud6, and Ud7 represent the voltage difference curves of the seven battery cells as time goes by at the experimental working conditions. As can be seen from the experimental results, that the difference voltage of the seven single cells exists from the beginning, and the voltage difference in both of the first phase and the second phase is within $10 \mathrm{mV}$. The cell voltage decreases gradually in the third phase, in which the voltage difference increases rapidly, reaching a maximum of $120 \mathrm{mV}$. The voltage difference under working condition 2 is shown in Fig. 9. 


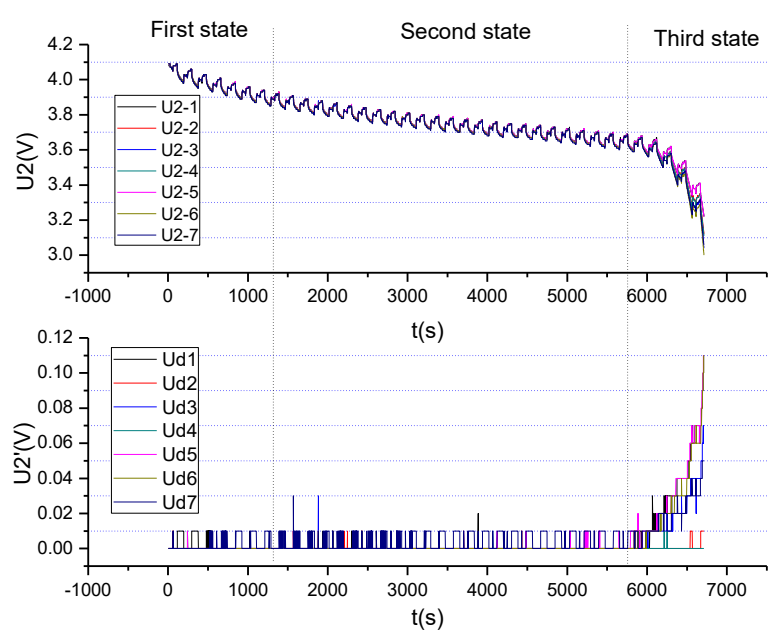

Fig. 9. Voltage difference under working condition 2

As can be known from Fig. 9, the signals of U2-1, U2-2, U2-3, U2-4, U2-5, U2-6, U2-7 represent the terminal voltage of 7 single cells under the working condition 2 . The signals of Ud1, Ud2, Ud3, Ud4, Ud5, Ud6, Ud7 in the second part of the figure represent the voltage difference curves of the seven battery cells under the experimental working condition 2 towards time. As can be seen from experimental results, the discharge depth of the single-cycle of the operating condition 2 is greater than the single-mode cycling discharge depth of the operating condition 1, and the voltage difference of the seven single cells is greater in the first stage. The voltage difference of the two stages is still within $10 \mathrm{mV}$. However, the battery cell difference increases in the overall discharging process in the third stage, as the battery cell voltage drops rapidly. Meanwhile, the battery cell voltage difference under the working condition 3 is shown in Fig. 10.
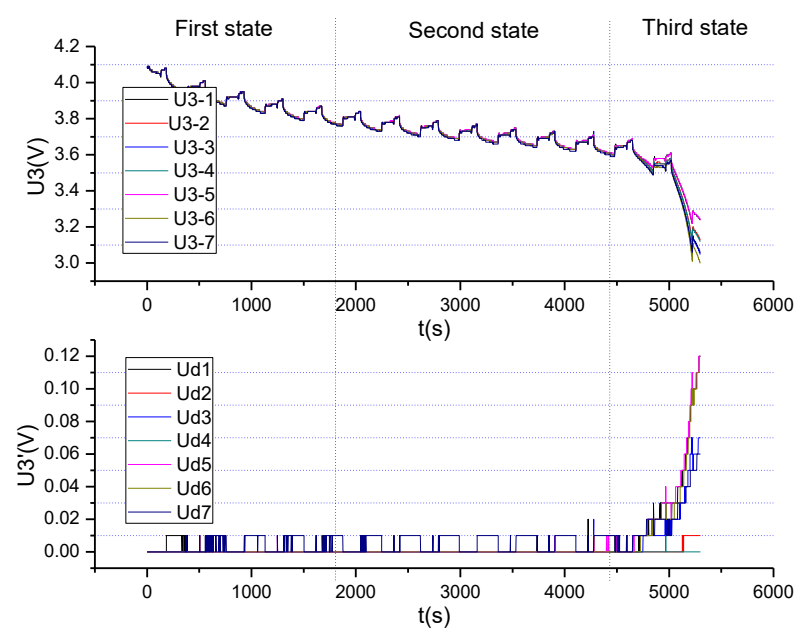

Fig. 10. Voltage difference under working condition 3

The signals of U3-1, U3-2, U3-3, U3-4, U3-5, U3-6, U3-7 in Fig. 10 represents the seven single battery cell voltages under the experimental working condition 3 . Ud1, Ud2, Ud3, Ud4, Ud5, Ud6, Ud7 in the second part of the figure represent the voltage difference curves of the seven battery cells under the working condition 3 . As can be seen from the experimental results, the single-cycle discharge depth of the operating condition 3 is the largest compared with the first two operating conditions, and the voltage difference of the seven single cells does not change in the first phase and the second phase, which has the similar changing laws compared with other researches [65][66]. In the third stage, the voltage difference increase is greater than the first two working conditions with the rapid decline of the cell voltages. Comparing the analysis of working condition 1,2 and 3, there is a voltage difference increase for the first and then for the second phase decreases gradually. The rising trend of the difference curve is increased gradually for the overall voltage of the third phase. The voltage difference between the cells increases along with the voltage drop at the end of the traditional working conditions. The battery cell inconsistency degree is proportional to the discharge depth of the charge-discharge cycle.

\section{Conclusions}

Aiming at the online power state evaluation of the lithium battery packs, the coupling relationship description of the external measurable parameters and the reliable grading power state evaluation are carried out to construct the coupling relationship model between the parameters under complex working conditions. As for the power state evaluation of the complex internal structure, the credibility calculation and combination rule selection are investigated to construct the equilibrium evaluation model by using the information fusion strategy. The knowledge base construction and the correction algorithm design eliminates the estimation errors caused by the environmental conditions. Based on the previous research work, this research is focused on the differences and mathematical characterization between the battery cells in the lithium battery pack, which provides state monitoring theory support for the subsequent battery packing work safety. Furthermore, the power state evaluation model is constructed by using the evidence theory by using the combined structure, in which the environmental influence factor and correction strategy are merged together to realize the complementary effect. The power state evaluation accuracy is improved for the lithium battery packs, which also provide new ideas for solving its safety problems in the automotive, aerospace and mobile base stations. The proposed power state evaluation method is verified by using the typical seven battery cell experimental samples. Subsequent verification experiments and method improvements for more 
complex cascades of battery cells will be carried out in the follow-up research process.

\section{Acknowledgments}

The work was supported by National Natural Science Foundation of China (No. 61801407), China Scholarship Council (No. 201908515099), Sichuan Province Science and Technology Support Program (No. 19ZDYF1098, 2019JDTD0019, 2019YFG0427, 2018GZ0390), Scientific Research Fund of Sichuan (No. 17ZB0453), Teaching Research Project (18lzx665, 18gjzx11, 18xnsu12). Thanks to the sponsors. CF would like to express his gratitude to PALS for its support.

\section{References}

1. Ren, H.B., et al., Design and implementation of a battery management system with active charge balance based on the SOC and SOH online estimation. Energy, 2019. 166: p. 908-917.

2. Zhang, C.P., et al., Study on battery pack consistency evolutions and equilibrium diagnosis for serial- connected lithium-ion battery cells. Applied Energy, 2017. 207: p. 510-519.

3. Lai, X.Q., Dongdong; Zheng, Yuejiu; Ouyang, Minggao; Han, Xuebing; Zhou, Long, A rapid screening and regrouping approach based on neural networks for large-scale retired lithium-ion cells in second-use applications. Journal of Cleaner Production, 2019. 213: $p$. 776-791.

4. Mai, W.J., M. Yang, and S. Soghrati, A particle-resolved 3D finite element model to study the effect of cathode microstructure on the behavior of lithium ion battery cells. Electrochimica Acta, 2019. 294: p. 192-209.

5. Mao, C.Y., et al., Balancing formation time and electrochemical performance of high energy lithium-ion battery cells. Journal of Power Sources, 2018. 402: p. 107-115.

6. Hu, X.S., S.E. Li, and Y.L. Yang, Advanced Machine Learning Approach for Lithium-Ion Battery State Estimation in Electric Vehicles. leee Transactions on Transportation Electrification, 2016. 2(2): p. 140-149.

7. Misyris, G.S., et al., State-of-Charge Estimation for Li-lon Battery cells: A More Accurate Hybrid Approach. leee Transactions on Energy Conversion, 2019. 34(1): p. 109-119.
8. Wang, J.Q., et al., A nanoporous sulfurbridged hexaazatrinaphthylene framework as an organic cathode for lithium ion battery cells with well-balanced electrochemical performance. Chemical Communications, 2018. 54(55): p. 76817684.

9. Ouyang, Q., et al., SOC Estimation-Based Quasi-Sliding Mode Control for Cell Balancing in Lithium-Ion Battery Packs. leee Transactions on Industrial Electronics, 2018. 65(4): p. 3427-3436.

10. Zhang, Z.L., et al., SOC Estimation of Lithium-lon Battery Pack Considering Balancing Current. leee Transactions on Power Electronics, 2018. 33(3): p. 22162226.

11. Ouyang, Q., et al., Optimal Cell-to-Cell Balancing Topology Design for Serially Connected Lithium-Ion Battery Packs. leee Transactions on Sustainable Energy, 2018. 9(1): p. 350-360.

12. Cai, L., et al., An evolutionary framework for lithium-ion battery state of health estimation. Journal of Power Sources, 2019. 412: p. 615-622.

13. Knap, V., et al., Self-balancing feature of Lithium-Sulfur battery cells. Journal of Power Sources, 2017. 372: p. 245-251.

14. Wang, Z.K., et al., State of health estimation of lithium-ion battery cells based on the constant voltage charging curve. Energy, 2019. 167: p. 661-669.

15. Li, S.X., et al., Fractional-order modeling and SOC estimation of lithium-ion battery considering capacity loss. International Journal of Energy Research, 2019. 43(1): p. 417-429.

16. Cui, X.D., et al., A Novel Active Online State of Charge Based Balancing Approach for Lithium-Ion Battery Packs during Fast Charging Process in Electric Vehicles. Energies, 2017. 10(11).

17. Wang, S.L., et al., Open circuit voltage and state of charge relationship functional optimization for the working state monitoring of the aerial lithium-ion battery pack. Journal of Cleaner Production, 2018. 198: p. 1090-1104. 
18. Chiang, Y.H., et al., Development of $a$ converterless energy management system for reusing automotive lithium-ion battery applied in smart-grid balancing. Journal of Cleaner Production, 2017. 156: p. 750-756.

19. Wang, S.L., et al., A novel safety anticipation estimation method for the aerial lithium-ion battery pack based on the real-time detection and filtering. Journal of Cleaner Production, 2018. 185: p. 187-197.

20. Cui, X.D., et al., A Fast Multi-Switched Inductor Balancing System Based on a Fuzzy Logic Controller for Lithium-Ion Battery Packs in Electric Vehicles. Energies, 2017. 10(7).

21. Zhou, F.W. and Z.Y. Lv, $A$ novel charging/discharging balancing strategy for lithium battery pack based on mixed charging mode. leej Transactions on Electrical and Electronic Engineering, 2017. 12(4): p. 615-622.

22. Yang, L.Q., et al., Balancing the chemisorption and charge transport properties of the interlayer in lithium-sulfur battery cells. Journal of Materials Chemistry A, 2017. 5(24): p. 12506-12512.

23. Richardson, R.R., et al., Gaussian Process Regression for In Situ Capacity Estimation of Lithium-Ion Battery cells. leee Transactions on Industrial Informatics, 2019. 15(1): p. 127-138.

24. Aklalouch, M., et al., High-performance Liion Battery based on Cr-Substituted Lithium Manganite and Lithium Titanate Spinels: Influence of Mass Balance on its Electrochemistry. Energy Technology, 2017. 5(5): p. 725-731.

25. Ma, Z.Y., et al., A mechanism identification model based state-of-health diagnosis of lithium-ion battery cells for energy storage applications. Journal of Cleaner Production, 2018. 193: p. 379-390.

26. Lipu, M.S.H., et al., A review of state of health and remaining useful life estimation methods for lithium-ion battery in electric vehicles: Challenges and recommendations. Journal of Cleaner Production, 2018. 205: p. 115-133.
27. Zhang, Z.L., et al., A Hierarchical Active Balancing Architecture for Lithium-lon Battery cells. leee Transactions on Power Electronics, 2017. 32(4): p. 2757-2768.

28. Li, Y., et al., Random forest regression for online capacity estimation of lithium-ion battery cells. Applied Energy, 2018. 232: p. 197-210.

29. Zhao, L.H., Z.Y. Liu, and G.H. Ji, Lithium-ion battery state of charge estimation with model parameters adaptation using $\mathrm{H}$ infinity, extended Kalman filter. Control Engineering Practice, 2018. 81: p. 114-128.

30. Chirkov, Y.G., et al., Computer Simulation of an Electrode of Lithium-Ion Battery: Estimation of Ohmic Losses for ActiveMaterial Grains Covered by a Conducting Film. Russian Journal of Electrochemistry, 2018. 54(11): p. 970-978.

31. McCurlie, L., M. Preindl, and A. Emadi, Fast Model Predictive Control for Redistributive Lithium-Ion Battery Balancing. leee Transactions on Industrial Electronics, 2017. 64(2): p. 1350-1357.

32. Gao, Z.C., et al., State-of-Charge Estimation and Active Cell Pack Balancing Design of Lithium Battery Power System for Smart Electric Vehicle. Journal of Advanced Transportation, 2017.

33. Liu, D.T., et al., On-line life cycle health assessment for lithium-ion battery in electric vehicles. Journal of Cleaner Production, 2018. 199: p. 1050-1065.

34. Methekar, R., SOC estimation with thermal and charging rate consideration using dual filter approach for lithium-ion battery. Journal of Renewable and Sustainable Energy, 2018. 10(6).

35. Kasnatscheew, J., et al., A Tutorial into Practical Capacity and Mass Balancing of Lithium Ion Battery cells. Journal of the Electrochemical Society, 2017. 164(12): p. A2479-A2486.

36. Docimo, D.J. and H.K. Fathy, Multivariable State Feedback Control as a Foundation for Lithium-lon Battery Pack Charge and Capacity Balancing. Journal of the Electrochemical Society, 2017. 164(2): p. A61-A70. 
37. Campestrini, C., et al., Ageing of lithium-ion battery modules with dissipative balancing compared with single-cell ageing. Journal of Energy Storage, 2016. 6: p. 142-152.

38. Ali, M.U., et al., An Online Data-Driven Model Identification and Adaptive State of Charge Estimation Approach for Lithiumion-Battery cells Using the Lagrange Multiplier Method. Energies, 2018. 11(11).

39. Tao, X.Y., et al., Balancing surface adsorption and diffusion of lithiumpolysulfides on nonconductive oxides for lithium-sulfur battery design. Nature Communications, 2016. 7.

40. Wang, S.L., et al., Online dynamic equalization adjustment of high-power lithium-ion battery packs based on the state of balance estimation. Applied Energy, 2016. 166: p. 44-58.

41. Li, J.H. and M.S. Liu, SOC estimation for lithium battery cells based on the full parallel nonlinear autoregressive neural network with external inputs. Journal of Renewable and Sustainable Energy, 2018. 10(6).

42. Hu, X.S., et al., Co-Estimation of State of Charge and State of Health for Lithium-Ion Battery cells Based on Fractional-Order Calculus. leee Transactions on Vehicular Technology, 2018. 67(11): p. 10319-10329.

43. Hoque, M.M., M.A. Hannan, and A. Mohamed, Voltage equalization control algorithm for monitoring and balancing of series connected lithium-ion battery. Journal of Renewable and Sustainable Energy, 2016. 8(2).

44. Ma, Z.Y., R.X. Yang, and Z.P. Wang, A novel data-model fusion state-of-health estimation approach for lithium-ion battery cells. Applied Energy, 2019. 237: p. 836-847.

45. Chen, Z.H., et al., Particle filter-based stateof-charge estimation and remainingdischargeable-time prediction method for lithium-ion battery cells. Journal of Power Sources, 2019. 414: p. 158-166.

46. Wei, Z.B., et al., Comparative study of methods for integrated model identification and state of charge estimation of lithium- ion battery. Journal of Power Sources, 2018. 402: p. 189-197.

47. Wang, X.Y., X.Z. Wei, and H.F. Dai, Estimation of state of health of lithium-ion battery cells based on charge transfer resistance considering different temperature and state of charge. Journal of Energy Storage, 2019. 21: p. 618-631.

48. Couto, L.D., et al., State of health estimation for lithium ion battery cells based on an equivalent-hydraulic model: An iron phosphate application. Journal of Energy Storage, 2019. 21: p. 259-271.

49. Guo, P.Y., Z. Cheng, and L. Yang, A datadriven remaining capacity estimation approach for lithium-ion battery cells based on charging health feature extraction. Journal of Power Sources, 2019. 412: p. 442-450.

50. Wang, T.P., et al., Model-based unscented Kalman filter observer design for lithium-ion battery state of charge estimation. International Journal of Energy Research, 2018. 42(4): p. 1603-1614.

51. Wang, Y.J., et al., Power capability evaluation for lithium iron phosphate battery cells based on multi-parameter constraints estimation. Journal of Power Sources, 2018. 374: p. 12-23.

52. Wang, S.L., et al., An integrated online adaptive state of charge estimation approach of high-power lithium-ion battery packs. Transactions of the Institute of Measurement and Control, 2018. 40(6): p. 1892-1910.

53. Wang, S.L., et al., Adaptive State-of-Charge Estimation Method for an Aeronautical Lithium-ion Battery Pack Based on a Reduced Particle-unscented Kalman Filter. Journal of Power Electronics, 2018. 18(4): p. 1127-1139.

54. Ning, B., et al., Adaptive sliding mode observers for lithium-ion battery state estimation based on parameters identified online. Energy, 2018. 153: p. 732-742.

55. Peng, S.M., et al., An adaptive state of charge estimation approach for lithium-ion series-connected battery system. Journal of Power Sources, 2018. 392: p. 48-59. 
56. Park, J., et al., Battery State Estimation Algorithm for High-Capacity Lithium Secondary Battery for EVs Considering Temperature Change Characteristics. Journal of Electrical Engineering \& Technology, 2018. 13(5): p. 1927-1934.

57. Tang, X.P., et al., $A$ fast estimation algorithm for lithium-ion battery state of health. Journal of Power Sources, 2018. 396: p. 453-458.

58. Salameh, M., et al., Thermal State of Charge Estimation in Phase Change Composites for Passively Cooled Lithium-Ion Battery Packs. leee Transactions on Industry Applications, 2018. 54(1): p. 426-436.

59. Shen, P., et al., The Co-estimation of State of Charge, State of Health, and State of Function for Lithium-lon Battery cells in Electric Vehicles. leee Transactions on Vehicular Technology, 2018. 67(1): p. 92103.

60. Ruan, H.J., et al., Online estimation of thermal parameters based on a reduced wide-temperature-range electro-thermal coupled model for lithium-ion battery cells. Journal of Power Sources, 2018. 396: p. 715-724.

61. Yuan, C.C., et al., State-of-Charge Estimation of Lithium-ion Battery Based on a Novel Reduced Order Electrochemical Model. International Journal of Electrochemical Science, 2018. 13(1): p. 1131-1146.

62. Ye, M., et al., $A$ double-scale and adaptive particle filter-based online parameter and state of charge estimation method for lithium-ion battery cells. Energy, 2018. 144: p. 789-799.

63. Yang, Z., D. Patil, and B. Fahimi, Online Estimation of Capacity Fade and Power Fade of Lithium-Ion Battery cells Based on Input-Output Response Technique. leee Transactions on Transportation Electrification, 2018. 4(1): p. 147-156.

64. Yang, J.F., et al., Online state-of-health estimation for lithium-ion battery cells using constant-voltage charging current analysis. Applied Energy, 2018. 212: p. 1589-1600.
65. Yang, D., et al., A novel Gaussian process regression model for state-of-health estimation of lithium-ion battery using charging curve. Journal of Power Sources, 2018. 384: p. 387-395.

66. Xie, J.L., J.C. Ma, and K. Bai, Enhanced Coulomb Counting Method for State-ofCharge Estimation of Lithium-ion Battery cells based on Peukert's Law and Coulombic Efficiency. Journal of Power Electronics, 2018. 18(3): p. 910-922. 